\title{
Bronchiolitis clinical practice guidelines implementation: surveillance study of hospitalized children in Jordan
}

\author{
Samah Awad, ${ }^{1}$ Rawan Hatim, ${ }^{1}$ Yousef Khader, ${ }^{2}$ Mohammad Alyahya, ${ }^{3}$ Nada Harik, ${ }^{4}$ Ahmad Rawashdeh, ${ }^{1}$ \\ Walaa Qudah, ${ }^{1}$ Ruba Khasawneh, ${ }^{5}$ Wail Hayajneh, ${ }^{1}$ Dawood Yusef ${ }^{1}$ \\ ${ }^{1}$ Department of Pediatrics and Neonatology, Faculty of Medicine, Jordan University of Science and Technology, Irbid, Jordan \\ ${ }^{2}$ Department of Public Health and Community Medicine, Faculty of Medicine, Jordan University of Science and Technology, \\ Irbid, Jordan \\ ${ }^{3}$ Department of Health Management and Policy, Faculty of Medicine, Jordan University of Science and Technology, Irbid, \\ Jordan
}

${ }^{4}$ Department of Pediatrics, School of Medicine and Health Sciences, George Washington University, Washington DC, USA ${ }^{5}$ Department of Diagnostic Radiology and Nuclear Medicine, Faculty of Medicine, Jordan University of Science and Technology, Irbid, Jordan

Introduction: Bronchiolitis is a leading cause of hospital admissions and death in young children. Clinical practice guidelines (CPG) to diagnose and manage bronchiolitis have helped healthcare providers to avoid unnecessary investigations and interventions and to provide evidence-based treatment. Aim of this study is to determine the effect of implementing CPG for the diagnosis and management of bronchiolitis in a tertiary hospital in Jordan.

Methods: The study compared children (age <24 months) diagnosed with bronchiolitis and who required admission to King Abdullah University Hospital in Irbid during the winter of 2017 (after CPG implementation) and age-matched children admitted in the winter of 2016. The proportion of patients receiving diagnostic tests and treatments in the two groups were compared.

Results: Eighty-eight and 91 patients were diagnosed with bronchiolitis before and after CPG implementation, respectively. Respiratory syncytial virus rapid antigen detection testing decreased after CPG implementation [n=64 $(72.7 \%)$ vs $\mathrm{n}=46(50.5 \%), \mathrm{p}=0.002]$. However, there was no significant change in terms of other diagnostic tests. The use of nebulized salbutamol $[\mathrm{n}=44(50 \%)$ vs $\mathrm{n}=29(31.9 \%), \mathrm{p}=0.01]$, hypertonic saline $[\mathrm{n}=39(44.3 \%)$ vs $\mathrm{n}=8(8.8 \%)$, $\mathrm{p}<0.001]$, and inappropriate antibiotics $[\mathrm{n}=31(35.2 \%)$ vs $\mathrm{n}=15(16.5 \%), \mathrm{p}=0.004]$ decreased after CPG implementation. There was no difference in mean LOS (standard deviation; SD) between the pre- and post-CPG groups [3.5 (2) vs 4 (3.4) days, $\mathrm{p}=0.19$ ]. The mean cost of stay (SD) was 449.4 (329.1) US dollars for pre-CPG compared to 507.3 (286.1) US dollars for the post-CPG group ( $\mathrm{p}=0.24)$.

Conclusion: We observed that the implementation of CPG for bronchiolitis diagnosis and management helped change physicians' behavior toward evidence-based practices. However, adherence to guidelines must be emphasized to improve practices in developing countries, focusing on the rational use of diagnostic testing, and avoiding use of unnecessary medications when managing children with a diagnosis of bronchiolitis.

Key words: Bronchiolitis; clinical practice guidelines; developing countries; implementation; Jordan.

Correspondence: Samah Awad, Associate Professor, Pediatric Pulmonology, Department of Pediatrics and Neonatology, Faculty of Medicine, Jordan University of Science and Technology, P.O. Box 3030, Irbid 22110, Jordan. Tel. +962.2.7200600 Ext. 40663. E-mail: smawad@just.edu.jo

Contributions: All the authors contributed sufficiently to this study. SA, YK, MA, NH, study concept and design; RH, AR, WQ, RK, data acquisition; SA, YK, MA, NH, WH, DY, data analysis/interpretation; SA, YK, MA, NH, WH, DY, manuscript drafting. All the authors revised the manuscript critically for important intellectual content. All the authors have read and approved the final version of the manuscript and agreed to be accountable for all aspects of the work.

Conflict of interest: The authors declare that they have no competing interests, and all authors confirm accuracy.

Funding: This research did not receive any specific grant from funding agencies in the public, commercial, or not-for-profit sectors. Availability of data and materials: Data are available upon request from corresponding author.

Ethics approval and consent to participate: Ethical approval for the clinical surveillance of children hospitalized with ARI was obtained from the Institutional Review Board of Jordan University of Science and Technology. Caregivers of children consented to participate in the surveillance study. 


\section{Introduction}

Bronchiolitis is a leading cause of hospital admissions and death in young children worldwide, resulting in a substantial burden on healthcare services [1]. Bronchiolitis-associated morbidity and mortality are predominantly reported in developing countries [2]. In Jordan, bronchiolitis caused by respiratory syncytial virus (RSV) and other viruses are a major cause of respiratory tract infections in hospitalized children $[3,4]$.

The diagnosis of bronchiolitis is clinical, as it is based on history and physical examination; routine diagnostic or radiographic testing is not required $[5,6]$. Regarding the management of bronchiolitis, there is no role for bronchodilators (salbutamol) [7], epinephrine, or corticosteroids [8]. Antibiotics are not indicated unless concomitant bacterial infection is present [6].

Clinical practice guidelines (CPG) to diagnose and manage bronchiolitis have been issued in many countries $[5,9,10]$. Although a lack of compliance with CPG and variations in practice in managing inpatient bronchiolitis have been reported [11-13], implementing CPG has helped healthcare providers to reduce variations in practice, providing evidence-based treatment, the avoidance of unnecessary investigations and interventions, and the appropriate utilization of healthcare resources [14-18].

There are no Jordanian national guidelines for diagnosing and managing bronchiolitis. Jordanian physicians rely on published international guidelines for managing pediatric bronchiolitis. Consequently, there are variations in healthcare planning and the degree of use of diagnostic and therapeutic measures in bronchiolitis management. To our knowledge, no study has addressed the effect of implementing international guidelines for inpatient management of bronchiolitis in developing countries including Jordan. Thus, we conducted this study to determine the effect of implementing international guidelines on the diagnosis and management of bronchiolitis in an academic-based hospital in Jordan which could help in guiding physicians toward evidence based medical practice and optimizing the utility of health resources.

\section{Methods}

\section{Study design and setting}

Clinical surveillance of children admitted with acute respiratory infection (ARI) to King Abdullah University Hospital (KAUH) was conducted during the 3-month winter period (January to March) of 2016 [4] and 2017 (data unpublished). CPG for bronchiolitis diagnosis and management were implemented in the pediatric department of KAUH during the summer of 2016. We studied the effect of implementing CPG on diagnostic testing and management of children $<24$ months who had a diagnosis of bronchiolitis and required admission during the winter of 2017 compared to their counterparts admitted before the implementation of CPG in 2016 by comparing the proportions of patients receiving diagnostic tests and treatments. Winter is the peak of ARI/bronchiolitis in Jordan [3]. Diagnostic testing used in the methodology of the surveillance studies in 2016 and 2017 was not included in this study or used for comparison. Ethical approval for the ARI surveillance was given by the Institutional Review Board of Jordan University of Science and Technology.

$\mathrm{KAUH}$ is an academic-based hospital affiliated with Jordan University of Science and Technology and is a tertiary medical center in the city of Irbid, Northern Jordan. It has a pediatric ward and patients are cared for by pediatric specialists and subspecialists. Patients are admitted through the emergency department, outpatient clinics, or referred from other hospitals.

\section{Study population}

We selected children who had a clinical diagnosis of bronchiolitis among patients with ARI. Demographic and detailed clinical data of patients were collected on admission by trained pediatric residents. Data included: age, gender, gestational age, smoking exposure, day care attendance, breast feeding history and presenting symptoms (fever, cough, rhinorrhea, wheezing, apnea, difficulty breathing and cyanosis). Physical examination findings at the time of presentation were recorded, including $\mathrm{SpO}_{2}$ readings, respiratory rate, axillary temperature, retractions, flaring, grunting and auscultatory findings: wheezing and crackles. Tachypnea was defined as a respiratory rate $\geq 60 /$ min for infants younger than 2 months, $\geq 50 / \mathrm{min}$ for infants 2 to 12 months old, and $\geq 40 / \mathrm{min}$ for children 12 to 60 months old. Severe disease was defined as any sign of respiratory distress (tachypnea, grunting, flaring, retractions, cyanosis), apnea, altered mental status, or $\mathrm{SpO}_{2}<90 \%$. Diagnostic and management plans were obtained from the electronic health record (EHR) system for each patient. Children who required intensive care unit admission or ventilator management, had chronic lung disease of prematurity, cardiac disease, chronic pulmonary disease, immunodeficiency, neurological disease, genetic abnormality or developed two or more previous episodes of wheezing attacks were excluded from the study (Figure 1).

Chest X-rays (CXRs) of all studied patients admitted during the study period in 2016 and 2017 were interpreted by a pediatric radiologist who was blinded to the diagnosis. Presence of a secondary bacterial diagnosis was documented when having bacterial urinary tract infection, bacteremia, or bacterial meningitis. Antibiotic use during hospitalization based on evidence of bacterial infection, including positive urine culture, positive blood culture, positive cerebrospinal fluid culture, acute otitis media, or possible pneumonia diagnosis clinically and by CXR (presence of consolidation interpreted by one radiologist), was considered as appropriate use.

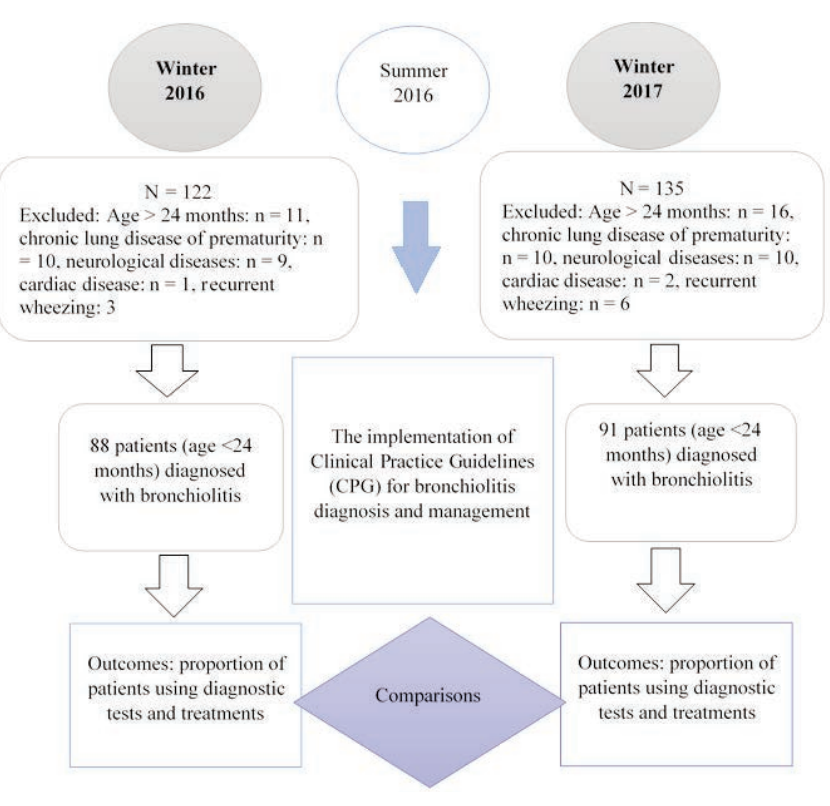

Figure 1. Flowchart of the study design. 


\section{Guidelines development and implementation}

Summary key points for diagnosing and managing bronchiolitis were developed from the American Academy of Pediatrics CPG for diagnosing and managing bronchiolitis (Table 1) [5]. They were drafted and revised by a pediatric pulmonologist and two pediatric infectious disease specialists. The CPG were made accessible online in the EHR system of the hospital with the assistance of the health information technology department. An order set was developed from the $\mathrm{CPG}$ and implemented in the EHR. A PowerPoint (Microsoft Corp., Redmond, WA) presentation with the content of the CPG was explained to practicing doctors including residents in an educational session in the pediatric department. Afterward, a demonstration of how to access the CPG and the order-set in the EHR was conducted for a mini-group of pediatric residents. Pediatric residents rotate in pediatric wards, emergency department and outpatient clinics, and all had received education. All pediatric patients at KAUH in pediatric wards, emergency department and outpatient clinics are managed by pediatric residents and specialists who were all included in CPG training. Investigators ensured all residents had received educational training via marking checklist for attendance. Some of the investigators were also clinical attending physicians supervising the residents caring for bronchiolitis patients during the 2016-2017 time period of the study. The CPG was implemented for patients admitted with bronchiolitis prior to the winter of 2017. A repeated educational session of bronchiolitis CPG content and order-set was delivered to the residents in 2017. Application of CPG in 2017 was randomly checked during clinical rounds, and periodically by interviewing the residents.

\section{Outcome measures}

The demographic and clinical characteristics of patients admitted with a bronchiolitis diagnosis were compared between 2017 (after CPG) and 2016 (before CPG). The outcome measure was the proportion of patients using diagnostic tests and treatments. Diagnostic tests included complete blood count (CBC), blood culture, urine analysis and culture, CXR, rapid RSV antigen testing, influenza reverse transcriptase-polymerase chain reaction testing. Treatments included nasal bulb suctioning, bronchodilators (scheduled nebulized salbutamol), single trial of salbutamol, nebulized hypertonic saline, inhaled and systemic steroids, antibiotics (inappropriate use), and chest physiotherapy. Difference in use of antibiotics between two groups was adjusted for the variables: prematurity, white blood cell count (WBC), CXR findings of consolidation and atelectasis, and secondary bacterial diagnosis. Secondary outcomes were the mean length of hospital stay (LOS) and admission cost.

\begin{abstract}
Statistical analysis
Statistical analysis was conducted using SPSS version 20 (IBM, Armonk, NY, USA). Data are described using means (standard deviation (SD)) for continuous variables and numbers and percentages for categorical variables. The differences in the means of continuous variables between pre-CPG and post-CPG implementation were analyzed using the independent $t$-test and the differences between proportions were analyzed using the Chi-square test. The difference in the use of antibiotics between the two time periods was analyzed using binary logistic regression to adjust for important variables. A p of $<0.05$ was considered statistically significant.
\end{abstract}

\section{Results}

\section{Demographic and clinical characteristics}

A total of 88 and 91 patients were diagnosed with bronchiolitis before and after CPG implementation, respectively. No significant differences were found in demographic data between both groups as shown in Table 2. Regarding the clinical presentation (Table 2), the post-CPG group had significantly greater occurrences of difficulty breathing, apnea, hypoxia $\left(\mathrm{SpO}_{2}<94 \%\right)$, and requirement for oxygen supplementation. Retraction and flaring occurred more significantly in the pre-CPG group. Severe disease was more frequent in the post-CPG group ( $\mathrm{p}=0.009)$ (Table 2$)$.

\section{LOS and cost}

There was no difference in mean LOS (SD) between the pre- and post-CPG groups [3.5 (2) vs 4 (3.4) days, $\mathrm{p}=0.19$ ]. The mean cost of stay (SD) was 318.7 (233.4) Jordanian dinars (JD) (449.4 (329.1) US dollars) for pre-CPG compared to 359.8 (202.9) JD (507.3 (286.1) US dollars) for the post-CPG group $(\mathrm{p}=0.24)$ (Table 2 ).

\section{Diagnostic testing}

The use of RSV rapid antigen detection declined significantly after CPG implementation ( $p=0.002)$ (Table 3$)$. The frequencies of $\mathrm{CBC}$, blood culture, urinalysis, urine culture, CXR, and influenza testing did not change after CPG (Table 3 ).

\section{Treatment measures}

There was a significant increase in the use of nasal bulb suctioning after CPG implementation $(\mathrm{p}<0.001)$ (Table 4). Use of nebulized salbutamol and hypertonic saline decreased in the post-CPG group compared to the pre-CPG group $(\mathrm{p}=0.014$ and $\mathrm{p}<0.001$, respectively) (Table 4). The rate of inhaled or systemic steroid use was low in both groups. Antibiotic use was not affected by CPG implementation. However, inappropriate antibiotic use (no evi-

Table 1. Summary of clinical practice guidelines for diagnosing and managing bronchiolitis.

- Diagnosis of bronchiolitis and assessment of disease severity should be based on history and physical examination
- Laboratory studies and chest radiography should not be routinely ordered for diagnosis of bronchiolitis
- Salbutamol should not be used
- Epinephrine should not be used
- Nebulized hypertonic saline $3 \%$ should not be routinely used, and may be considered for children with a diagnosis of bronchiolitis and have length of stay >72 hours
- Inhaled and systemic steroids should not be used
- Antibiotics should not be administered to patients with bronchiolitis unless there is concomitant bacterial infection
- Chest physiotherapy should not be used
- Consider nasal suctioning in infants with respiratory distress due to nasal blockage with secretions
- Nasogastric or intravenous fluids should be administered for patients who cannot tolerate oral hydration


dence of bacterial infection or pneumonia) reduced significantly after CPG ( $\mathrm{p}=0.004)$ (Table 4).

After adjustment for factors, including prematurity, WBC $>15,000$, presence of CXR findings (atelectasis and consolidation), and having a secondary bacterial diagnosis, a significant decline in the use of antibiotics was noted in the post-CPG group compared to the pre-CPG group (odds ratio: $0.2,95 \%$ confidence interval: $0.1-0.6, p=0.003)$. $\mathrm{WBC}>15,000$ and secondary bacterial diagnosis were significantly associated with antibiotic use among the tested variables in the regression model $(\mathrm{p}<0.001$ and $\mathrm{p}=0.003$, respectively) (Table 5).

\section{Discussion}

Our study showed that implementing international CPG and making them accessible in the EHR system was helpful in minimizing the unnecessary use of medications for managing inpatient bronchiolitis in a tertiary medical center in Jordan. However, no change was noted in the ordering diagnostic tests $(\mathrm{CBC}$, blood culture, urine culture, CXR) except for RSV testing which declined after CPG implementation. Our results were similar to those of a multicenter cohort study in the US in which CPG did not significantly reduce the ordering of tests unless they were used for longer periods [17].

RSV antigen detection testing was successfully reduced after CPG. However, half of the patients were tested for RSV after the intervention, a value considered higher than previously reported [19]. Routine viral testing to detect the etiology of bronchiolitis is not recommended based on evidence but it can assist in the cohorting of patients and avoiding unnecessary antibiotic use [20]. Influenza testing frequency was not changed, possibly explained by the fact that the period of our study coincided with the influenza epidemic season in Jordan [21], where antiviral therapy is available and can be helpful [22].

Our study showed that doctors leaned towards ordering diagnostic tests and the evaluation of serious bacterial infection which has been associated with increased LOS and antibiotic overuse and a low-positive yield in patients with bronchiolitis [23]. In our study, WBC count was significantly associated with antibiotic use in the logistic regression analysis. Almost all patients in both preand post-CPG groups had CBC testing which highlights the lack of specific indication and a routine practice that was not changed after CPG.

Physicians were not adherent to the recommendation of not ordering CXR routinely, as CXR was ordered in all cases of admitted bronchiolitis before and after CPG. Patients after CPG tended to have more severe disease presentations which may partly explain this finding. Our result is similar to that of a study low- and middle-income countries (LMIC) that demonstrated no change in CXR use after CPG implementation [15]. Previous research in developed countries[14,16,24] showed a significant decrease in CXR use after CPG. Factors leading to overuse of CXR by physicians in managing bronchiolitis in developing or LMIC countries need to be studied. Perhaps physicians might not feel confident relying on clinical assessment for diagnosing bronchiolitis, or they are concerned about missing cases of bacterial pneumonia cases; for example, children in Jordan are not vaccinated against streptococcus pneumonia. When residents were asked about reasons of ordering tests in patients with bronchiolitis, responses included: fear of missing another diagnosis as urinary tract infection in a febrile child below the age of 2 years or pneumonia in a child with respiratory symptoms, ordering all the labs earlier is better than waiting for the results if another diagnosis is suspected later in the course of the illness and sometimes lack of awareness of the sequences of ordering unnecessary tests.

Implementing CPG was helpful in minimizing the inappropriate use of antibiotics. Overuse of antibiotics is a major health issue, and needs to be targeted to lessen cost, side effects, and antimicrobial resistance [25]. We determined that cases requiring antibiotics were based on evidence, including the presence of secondary bacterial diagnosis (positive bacterial cultures: urinary tract infection,

Table 2. Comparison of demographic and clinical characteristics of patients pre- and post- clinical practice guidelines.

\begin{tabular}{|c|c|c|c|}
\hline Variable & Pre-CPG & Post- CPG & $\mathbf{p}$ \\
\hline Age (months), mean (SD) & $7.3(5.6)$ & $6.6(5.1)$ & 0.35 \\
\hline $\begin{array}{l}\text { Sex } \\
\text { Female } \\
\text { Male }\end{array}$ & $\begin{array}{l}31(35.2) \\
57(64.8)\end{array}$ & $\begin{array}{l}29(32.2) \\
61(67.8)\end{array}$ & 0.67 \\
\hline $\begin{array}{l}\text { Gestational age } \\
\text { Premature } \\
\text { Full term }\end{array}$ & $\begin{array}{l}13(14.8) \\
75(85.2)\end{array}$ & $\begin{array}{l}14(15.4) \\
77(84.6)\end{array}$ & 0.91 \\
\hline Smoking exposure & $52(59.1)$ & $56(61.5)$ & 0.74 \\
\hline Day care attendance & $13(14.8)$ & $14(15.4)$ & 0.91 \\
\hline Breast feeding & $70(79.5)$ & $62(68.1)$ & 0.08 \\
\hline Axillary temperature $>38^{\circ} \mathrm{C}$ & $44(60.3)$ & $42(46.2)$ & 0.07 \\
\hline Rhinorrhea & $59(67)$ & $66(72.5)$ & 0.42 \\
\hline Cough & $80(90.9)$ & $87(95.6)$ & 0.21 \\
\hline Wheezing & $54(61.4)$ & $58(63.7)$ & 0.74 \\
\hline Apnea & $3(3.4)$ & $25(27.5)$ & $<0.001^{*}$ \\
\hline Difficulty breathing & $67(76.1)$ & $80(87.9)$ & $0.04^{*}$ \\
\hline Cyanosis & $11(12.5)$ & $20(22)$ & 0.09 \\
\hline Tachypnea & $60(68.2)$ & $50(54.9)$ & 0.07 \\
\hline $\begin{array}{l}\mathrm{SpO}_{2} \\
>94 \\
90-94 \\
<90\end{array}$ & $\begin{array}{l}60(68.2) \\
12(13.6) \\
16(18.2)\end{array}$ & $\begin{array}{l}41(45.1) \\
19(20.9) \\
31(34.1)\end{array}$ & $0.007 *$ \\
\hline Retractions & $45(51.1)$ & $31(34.1)$ & $0.02^{*}$ \\
\hline Flaring & $35(39.8)$ & $14(15.4)$ & $<0.001^{*}$ \\
\hline Grunting & $15(17)$ & $9(9.9)$ & 0.16 \\
\hline Wheezing & $47(53.4)$ & $44(48.4)$ & 0.50 \\
\hline Crackles & $36(40.9)$ & $5(5.5)$ & $<0.001^{*}$ \\
\hline Severe disease & $65(73.9)$ & $81(89)$ & $0.009 *$ \\
\hline $\begin{array}{l}\text { Chest radiograph findings } \\
\text { Interstitial markings } \\
\text { Hyperinflation } \\
\text { Atelectasis } \\
\text { Peribronchial thickening } \\
\text { Consolidation } \\
\text { Normal radiographic findings }\end{array}$ & $\begin{array}{l}36(40.9) \\
25(28.4) \\
12(13.6) \\
45(51.1) \\
13(14.8) \\
30(34.1)\end{array}$ & $\begin{array}{l}34(38.2) \\
18(20.2) \\
22(24.7) \\
47(52.8) \\
12(13.3) \\
22(24.7)\end{array}$ & $\begin{array}{l}0.71 \\
0.20 \\
0.06 \\
0.82 \\
0.78 \\
0.17\end{array}$ \\
\hline $\begin{array}{l}\text { White blood count } \\
<15000 \\
>15000\end{array}$ & $\begin{array}{l}58(67.4) \\
28(32.6)\end{array}$ & $\begin{array}{l}68(74.7) \\
23(25.3)\end{array}$ & 0.29 \\
\hline Respiratory syncytial virus positive & $19(21.6)$ & $21(23.1)$ & 0.81 \\
\hline Secondary diagnosis & $25(28.4)$ & $37(40.7)$ & 0.09 \\
\hline $\begin{array}{l}\text { Length of stay, days } \\
<3 \\
>3\end{array}$ & $\begin{array}{l}54(61.4) \\
34(38.6)\end{array}$ & $\begin{array}{l}54(59.3) \\
37(40.7)\end{array}$ & 0.78 \\
\hline Length of stay (mean), days & $3.47(2)$ & $4.04(3.5)$ & 0.19 \\
\hline Total cost mean (SD), US\$ & $449.4(329.1)$ & $507.3(286.1)$ & 0.24 \\
\hline
\end{tabular}

Data presented as number and (\%); SD, standard deviation; * statistically significant at $\mathrm{p}<0.05$. 
meningitis, and bacteremia) or pneumonia in both groups. Other studies showed that CPG did not reduce the use of antibiotics in bronchiolitis $[15,17,24]$ possibly because of the presence of concomitant bacterial infection requiring antibiotics that was not identified.

In our study, CPG helped to decrease the use of nebulized salbutamol as in previous studies [14,24]. A trial of bronchodilator is not recommended considering evidence in managing bronchiolitis [5]. However, the use of a single salbutamol trial did not change after CPG. This could be because it was administered in the emergency department prior to admission or was given to address parents' dissatisfaction when their children were not getting any treatment. Reports indicate that a rationale for medication prescription by practitioners in bronchiolitis could be the expectation of parents [26].

The role of nebulized hypertonic saline in bronchiolitis is controversial [27]. The American Academy of Pediatrics recommends the use of nebulized hypertonic saline $(3 \%)$ in the inpatient setting for non-severe bronchiolitis as it may decrease LOS in cases where LOS exceeds 3 days $[5,28,29]$. Before CPG, there was an overuse of hypertonic saline in the study population, and this declined significantly after CPG.

Despite the increase in the proportion of patients that received appropriate diagnosis and treatment, there was no change in LOS after CPG as reported previously [15]. However, other confounding variables could have attributed to LOS such as disease severity. A higher number of patients in the post-CPG group were hypoxic and had a severe disease presentation which could have necessitated a relatively longer LOS to facilitate oxygen supplementation and appropriate care. Although the post-CPG group had a non-significantly higher rate of a secondary diagnosis than the pre-CPG group, this could have influ-

Table 3. Comparison of diagnostic tests used pre- and post-clinical practice guidelines implementation.

\begin{tabular}{lccc} 
Diagnostic testing & Pre-CPG & Post-CPG & p \\
Complete blood count & $86(97.7)$ & $91(100)$ & 0.15 \\
Blood culture & $54(61.4)$ & $50(54.9)$ & 0.38 \\
\hline $\begin{array}{l}\text { Urine analysis and culture } \\
\text { Chest radiography }\end{array}$ & $36(40.9)$ & $36(39.6)$ & 0.85 \\
& $88(100)$ & $91(100)$ & $\begin{array}{c}\text { No statistics } \\
\text { computed\# }\end{array}$ \\
\hline Respiratory syncytial virus & $64(72.7)$ & $46(50.5)$ & $0.002^{*}$ \\
Influenza testing & $40(45.5)$ & $37(40.7)$ & 0.51 \\
\hline
\end{tabular}

Data presented as number and (\%); CPG, clinical practice guidelines; \#number is equal in both groups; * ${ }^{*}$ tatistically significant at $\mathrm{p}<0.05$. enced LOS in order for the patient to receive required treatment.

The total cost of stay was not changed after CPG. Other nonhealthcare-related factors could have influenced the total cost such as the parents' preference for particular hospital accommodation services which have more than one option with variable expenses.

\section{Strengths and limitations}

Our study included patients with bronchiolitis who were enrolled based on clinical diagnosis not on discharge diagnosis codes as in other studies $[16,17]$. This reduced the possibility of missing or misdiagnosing cases of bronchiolitis and allowed the recognition of severe disease. Moreover, we determined the presence of comorbidities and excluded them from the study, as they could have increased the risk of severe disease and influenced management plan, LOS, and cost. Further, we identified the presence of concomitant bacterial illness and justified antibiotic use which helped in recognizing antibiotic overuse in our study.

Our study was limited by its small sample size and perhaps, extending the duration of patient enrollment would have validated our findings.

\section{Conclusions}

CPG for bronchiolitis diagnosis and management are useful in changing physicians' behavior toward evidence-based practices in this study which is the first to investigate the effect of implementing CPG on diagnosis and management of bronchiolitis in devel-

Table 4. Treatments of patients pre- and post-clinical practice guidelines implementation.

\begin{tabular}{lccc} 
Diagnostic testing & Pre-CPG & Post-CPG & p \\
Therapeutic intervention & Pre-CPG & Post-CPG & p \\
Nasal suctioning & $9(10.2)$ & $83(91.2)$ & $<0.001^{*}$ \\
\hline Scheduled salbutamol & $44(50)$ & $29(31.9)$ & $0.01^{*}$ \\
Salbutamol trial & $24(27.3)$ & $21(23.1)$ & 0.52 \\
\hline Nebulized hypertonic saline & $39(44.3)$ & $8(8.8)$ & $<0.001^{*}$ \\
Inhaled corticosteroid & $3(3.4)$ & $3(3.3)$ & 0.97 \\
\hline Systemic steroids & $8(9.1)$ & $5(5.5)$ & 0.35 \\
Antibiotics & $52(59.1)$ & $44(48.4)$ & 0.15 \\
\hline $\begin{array}{l}\text { Inappropriate antibiotic use } \\
\text { (no indication) }\end{array}$ & $31(35.2)$ & $15(16.5)$ & $0.004^{*}$ \\
Chest physiotherapy & $7(8.0)$ & $8(8.8)$ & 0.840 \\
\hline
\end{tabular}

Data presented as number and (\%); *statistically significant at $\mathrm{p}<0.05$.

Table 5. Comparison of antibiotic use pre- and post-clinical practice guidelines.

\begin{tabular}{|c|c|c|c|}
\hline & OR & 95\% confidence interval & $\mathbf{p}$ \\
\hline Antibiotic use (post-CPG vs pre-CPG) & 0.23 & $0.09,0.62$ & $0.003 *$ \\
\hline \multicolumn{4}{|l|}{ Variables affecting antibiotic use } \\
\hline Gestational age (premature vs. full term) & 1.10 & $0.26,4.62$ & 0.901 \\
\hline White blood cell count $\geq 15,000 \mathrm{v} v s<15,000$ ) & 4.23 & $1.62,11.05$ & $0.003 *$ \\
\hline Gender (female $v s$ male) & 1.39 & $0.54,3.58$ & 0.497 \\
\hline Chest radiograph finding (consolidation vs no consolidation) & 3.67 & $0.49,27.55$ & 0.207 \\
\hline Chest radiograph finding (atelectasis vs no atelectasis) & 1.68 & $0.58,4.88$ & 0.337 \\
\hline Secondary bacterial diagnosis & 248.21 & $28.85,2135.65$ & $0.000^{*}$ \\
\hline
\end{tabular}

Logistic regression analysis for variables could have affected antibiotic use in both groups of patients pre- and post-CPG; CPG, clinical practice guidelines; \#number is equal in both groups; *statistically significant at $\mathrm{p}<0.05$ 
oping countries that have healthcare systems with limited resources. These findings orient physicians and stakeholders in the health care sector in Jordan to the necessity of establishing and implementing CPG for managing inpatients with bronchiolitis. Barriers to implement CPG and adherence to minimizing unnecessary diagnostic testing and treatment need to be assessed for improving clinical practice and optimizing resource utilization.

\section{References}

1. Shi T, McAllister DA, O'Brien KL, Simoes EAF, Madhi SA, Gessner BD, et al. Global, regional, and national disease burden estimates of acute lower respiratory infections due to respiratory syncytial virus in young children in 2015: a systematic review and modelling study. Lancet 2017;390:946-58.

2. Nair H, Nokes DJ, Gessner BD, Dherani M, Madhi SA, Singleton $\mathrm{RJ}$, et al. Global burden of acute lower respiratory infections due to respiratory syncytial virus in young children: a systematic review and meta-analysis. Lance. 2010;375:1545-55.

3. Halasa N, Williams J, Faouri S, Shehabi A, Vermund SH, Wang L, et al. Natural history and epidemiology of respiratory syncytial virus infection in the Middle East: Hospital surveillance for children under age two in Jordan. Vaccine 2015;33:6479-87.

4. Awad S, Khader Y, Mansi M, Yusef D, Alawadin S, Qudah W, et al. Viral Surveillance of Children with Acute Respiratory Infection in Two Main Hospitals in Northern Jordan, Irbid, during Winter of 2016. J Pediatr Infect Dis 2020;15:1-10.

5. Ralston SL, Lieberthal AS, Meissner HC, Alverson BK, Baley JE, Gadomski AM, et al. Clinical practice guideline: The diagnosis, management, and prevention of bronchiolitis. Pediatrics 2014;134:e1474-502.

6. Florin TA, Plint AC, Zorc JJ. Viral bronchiolitis. Lancet 2017;389:211-24

7. Gadomski AM, Scribani MB. Bronchodilators for bronchiolitis. Cochrane Database Syst Rev 2014;2014:CD001266.

8. Fernandes RM, Bialy LM, Vandermeer B, Tjosvold L, Plint AC, Patel $\mathrm{H}$, et al. Glucocorticoids for acute viral bronchiolitis in infants and young children. Cochrane Database Syst Rev 2013;2013:CD004878.

9. Caffrey Osvald E, Clarke JR. NICE clinical guideline: bronchiolitis in children. Arch Dis Child Educ Pract Ed 2016;101:46-8.

10. Green RJ, Zar HJ, Jeena PM, Madhi SA, Lewis H. South African guideline for the diagnosis, management and prevention of acute viral bronchiolitis in children. South African Med J 2010;100:3205.

11. Christakis DA, Cowan CA, Garrison MM, Molteni R, Marcuse E, Zerr DM. Variation in inpatient diagnostic testing and management of bronchiolitis. Pediatrics 2005;115:878-84.

12. Elenius V, Bergroth E, Koponen P, Remes S, Piedra PA, Espinola JA, et al. Marked variability observed in inpatient management of bronchiolitis in three Finnish hospitals. Acta Paediatr 2017;106:1512-8.

13. Macias CG, Mansbach JM, Fisher ES, Riederer M, Piedra PA, Sullivan AF, et al. Variability in inpatient management of children hospitalized with bronchiolitis. Acad Pediatr 2015;15:69-76.

14. Breakell R, Thorndyke B, Clennett J, Harkensee C. Reducing unnecessary chest $\mathrm{X}$-rays, antibiotics and bronchodilators through implementation of the NICE bronchiolitis guideline. Eur J Pediatr 2018; $177: 47-51$.

15. Henao-Villada R, Sossa-Briceño MP, Rodríguez-Martínez CE. Impact of the implementation of an evidence-based guideline on diagnostic testing, management, and clinical outcomes for infants with bronchiolitis. Ther Adv Respir Dis 2016;10:425-34.

16. McCulloh RJ, Smitherman SE, Koehn KL, Alverson BK. Assessing the impact of national guidelines on the management of children hospitalized for acute bronchiolitis. Pediatr Pulmonol 2014;49:688-94.

17. Mittal V, Hall M, Morse R, Wilson KM, Mussman G, Hain P, et al. Impact of inpatient bronchiolitis clinical practice guideline implementation on testing and treatment. J Pediatr 2014;165:570-6.e3.

18. Parikh K, Hall M, Teach SJ. Bronchiolitis management before and after the AAP guidelines. Pediatrics 2014;133:e1-7.

19. Emerson BL, Tenore C, Grossman M. An initiative to reduce routine viral diagnostic testing in pediatric patients admitted with bronchiolitis. Jt Comm J Qual Patient Saf 2018;44:751-6.

20. Harris JAS, Huskins WC, Langley JM, Siegel JD, Pediatric Special Interest Group of the Society for Healthcare Epidemiology of America. Health care epidemiology perspective on the October 2006 recommendations of the Subcommittee on Diagnosis and Management of Bronchiolitis. Pediatrics 2007;120:890-2.

21. Caini S, El-Guerche Séblain C, Ciblak MA, Paget J. Epidemiology of seasonal influenza in the Middle East and North Africa regions, 2010-2016: Circulating influenza A and B viruses and spatial timing of epidemics. Influenza Other Respir Viruses 2018;12:34452.

22. Rothberg MB, Fisher D, Kelly B, Rose DN. Management of influenza symptoms in healthy children: cost-effectiveness of rapid testing and antiviral therapy. Arch Pediatr Adolesc Med 2005; 159:1055-62.

23. Librizzi J, McCulloh R, Koehn K, Alverson B. Appropriateness of testing for serious bacterial infection in children hospitalized with bronchiolitis. Hosp Pediatr 2014;4:33-8.

24. Mittal V, Darnell C, Walsh B, Mehta A, Badawy M, Morse R, et al. Inpatient bronchiolitis guideline implementation and resource utilization. Pediatrics. American Academy of Pediatrics; 2014;133.

25. Llor C, Bjerrum L. Antimicrobial resistance: risk associated with antibiotic overuse and initiatives to reduce the problem. Ther Adv drug Saf. 2014;5:229-41.

26. Carande EJ, Cheung CR, Pollard AJ, Drysdale SB. Change in viral bronchiolitis management in primary care in the UK after the publication of NICE guideline. Thorax. 2018;

27. Harrison W, Angoulvant F, House S, Gajdos V, Ralston SL. Hypertonic saline in bronchiolitis and type i error: A trial sequential analysis. Pediatrics. American Academy of Pediatrics; 2018;142.

28. Zhang L, Mendoza-Sassi RA, Wainwright C, Klassen TP. Nebulised hypertonic saline solution for acute bronchiolitis in infants. Cochrane database Syst Rev. 2017;12:CD006458.

29. Wang Z, Li X, Sun A, Fu X. Efficacy of 3\% hypertonic saline in bronchiolitis: A meta-analysis. Exp Ther Med. Spandidos Publications; 2019;18.

Received for publication: 1 May 2020. Accepted for publication: 8 September 2020.

This work is licensed under a Creative Commons Attribution-NonCommercial 4.0 International License (CC BY-NC 4.0).

(C) Copyright: the Author(s), 2020

Licensee PAGEPress, Italy

Multidisciplinary Respiratory Medicine 2020; 15:673

doi:10.4081/mrm.2020.673 